\title{
Experimental studies of surface waves inside a cylindrical container
}

\author{
CUNBIAO LEE ${ }^{1} \uparrow$, HUAIWU PENG ${ }^{1}$, HUIJING YUAN ${ }^{1}$, \\ JIEZHI WU ${ }^{1}$, MINGDE ZHOU ${ }^{1}$ AND FAZLE HUSS AIN ${ }^{2}$
}

${ }^{1}$ State Key Laboratory for Turbulence and Complex Systems, Department of Mechanics and Aerospace Engineering, College of Engineering, Peking University, Beijing 100871, China

${ }^{2}$ Department of Mechanical Engineering, University of Houston, Houston, TX 77204 4006, USA

(Received 1 February 2010; revised 16 January 2011; accepted 22 January 2011; first published online 9 May 2011)

We experimentally investigate the dynamics of surface waves excited by oscillations from a cylindrical sidewall. Particle-imaging-velocimetry measurements with fluorescent particles were used to determine the flow patterns near the sidewall of the cylindrical fluid container and to identify the locations of the evolving airwater interfaces. The high-frequency wall oscillations created four jets that originate at the cylindrical sidewall. Four vortex streets shed from the jets propagate from the sidewall to the centre of the container and subsequently excite a low-frequency gravity wave. The interaction between this gravitational surface wave and the highfrequency capillary waves was found to be responsible for creating droplet splash at the water surface. This phenomenon was first described as 'Long-Xi' or 'dragon wash' in ancient China. The physical processes for generating the droplet ejection, including the circular capillary waves, azimuthal waves, streaming jets and low-frequency gravity waves, are described in this paper.

Key words: capillary waves, instability

\section{Introduction}

Parametrically driven surface waves were first observed in a vertically excited container by Faraday (1831). Miles \& Henderson (1990) revisited the phenomenon in detail. Sidewall forcing induced free-surface oscillations were experimentally observed in a tank with a free fluid surface and described as a spatial-resonance phenomenon (Huntley 1972; Mahony \& Smith 1972). Nonlinear wave interactions can also occur via non-resonant mode interaction between the short and long waves. Mahony \& Smith (1972) observed that standing surface waves inside water can also be excited by high-frequency acoustic waves.

The 'dragon wash' phenomenon found in China is related to spatial resonance. Typically, this phenomenon occurs in a water-filled circular bronze basin with two handles welded symmetrically on the flared-out brim. Rubbing the handles with both hands or with one hand produces standing waves on the free surface. Rubbing faster and harder amplifies the standing waves, causing the water in the basin to splash high 
into the air. Wang $(1993,2005)$ carried out experimental and theoretical works on the mechanical properties of dragon wash. He used a cylindrical container to stimulate the dragon wash basin with single-point, sidewall forcing from an electromagnetic actuator to simulate the rubbing. He found that a low-frequency axisymmetric wave could be generated by the high-frequency asymmetric vibrations applied to the sidewall of the circular container. Wei et al. (1997) did the first particle-imaging-velocimetry (PIV) measurements on the flow fields inside a water-filled glass cylinder with two-point excitation on its sidewall. They found a vortex ring under the free surface around the cylinder. Hsieh \& Denissenko (1998) studied shadowgraphs of the induced surface waves in a circular aluminium vessel and observed the small amplitude axisymmetric capillary waves. Liu et al. (2005) suggested that the vibration mechanism of the lowfrequency, large-amplitude, three-dimensional gravity waves was due to systematic resonance. Shen \& Hsieh (1993) obtained an exact solution for the linear problem of forced three-dimensional capillary-gravity waves in a circular container with a lateral prescribed harmonic forcing using Green's functions. Shen \& Yeh (1997) obtained a unique exact solution using Green's functions for the same linear problem with the Hocking's edge condition at the contact line, that is, the surface speed is proportional to the surface slope in the normal direction to the sidewall at the contact line. Sun, Shen \& Hsieh (1995), Chang (1999) and Chang \& Shen $(2000 a, b)$ developed a weakly nonlinear theory to explain the generation of the low-frequency axisymmetric wave in a circular geometry with lateral high-frequency forcing. Among others, Hsieh (1994, 1997, 2000), Hsieh \& Denissenko (1998) have also made important contributions to the dragon wash problem and identified the following three unresolved problems.

(i) What is the mechanism for the generation of the azimuthal capillary water waves on the wall at the water interface on the container?

(ii) What is the physical mechanism for the appearance of the jumping droplets?

(iii) Why are the low-frequency, three-dimensional gravity waves excited by the high-frequency external forcing?

The third problem is the main focus of the present paper which describes oscillationinduced jets, jet instability and jet-induced sloshing by lateral single-point forcing of the sidewall of a water-filled container (Peng, Wang \& Lee 2005; Peng et al. 2006, 2008; Peng \& Lee 2009). The interactions between the free surface and submerged vortical flows have been investigated by many researchers due to their fundamental importance and practical applications. The horizontal jet induced sloshing motion was first observed by Okamoto \& Hagiwara (1991). Many experimental, numerical and theoretical works have been undertaken since then. Some numerical simulations of self-induced sloshing (Takizawa, Koshizuka \& Kondo 1992; Saeki et al. 1997) have agreed well with experimental data, suggesting that the self-induced sloshing mainly depends on the inlet jet fluctuations. Several experimental and theoretical models have been proposed for self-induced sloshing excited by a horizontally injected jet (Okamoto \& Hagiwara 1991; Fukaya, Madarame \& Okamoto 1996; Saeki, Madarame \& Okamoto 2001). Hu et al. (1999) used PIV measurements to quantitatively investigate self-sustained sloshing in a circulating flow. The near-wall jets and the breaking waves resulting from resonant excitation of a circular basin was also investigated by Royon-Lebeaud, Hopfinger \& Cartellier (2007), in addition to the work by Faltinsen, Rognebakke \& Timokha $(2006 a, b)$ also observed these phenomena, but in a square tank. For high-frequency forcing, Sharipov \& Kremer (1999) and Gavrilyuk, Lukovsky \& Timokha (2004) studied the two-dimensional vibroequilibria of a confirmed incompressible fluid volume harmonically forced at high frequency. 
This paper describes new mechanisms causing the emergence of low-frequency, three-dimensional gravity waves excited by high-frequency, external forcing in cylindrical containers. The generation mechanisms for the three-dimensional gravity waves are further illustrated by complementary experiments conducted in a circular cylindrical container. Image analyses and PIV flow field measurements of the surface waves were used to study the evolution of the wave motion as well as the water surface velocities and the vortex generation. Section 2 describes the experimental configuration and procedure. Section 3 describes the circular and azimuthal capillary waves that occur with the high-frequency excitation. Section 4 describes the streaming vortices and droplet ejection while $\S 5$ describes the jet-like flows and detailed generation scenarios of the low-frequency gravity waves, which is the focus of this paper. The discussion and conclusions are presented in $\S 6$ and $\S 7$.

\section{Experimental methods}

The experimental apparatus included a circular glass tank with a vertical sidewall with inner diameter, height, and wall thickness of 210, 220 and $3 \mathrm{~mm}$ (figure 1). The container was filled with de-ionized water to various depths corresponding to different excitation frequencies. The excitation forcing was provided by means of the wall acceleration. The experimental setup allowed use of surface flow shadowgraphs, a method similar to that used by Hsieh \& Denissenko (1998).

To reduce the effect of uneven lighting on the image and to extract useful information on the surface waves, each image was subtracted from a background image taken without external forcing. The shadowgraph method was most effective for small amplitude waves. The droplet ejection regime had relatively large wave amplitudes and curvature, leading to fuzzy images. A statistical method was used to determine the droplet ejection threshold at various forcing frequencies. These results show that the forced surface waves exhibit three distinct wave patterns, circular capillary waves, azimuthal waves and pre-ejection waves. Each pattern was separated by a transition wave pattern as will be explained in the results.

A fluorescent PIV system was used to measure the velocity fields in the horizontal and vertical planes inside the glass container (Tower \& Reeves 1999). A spherical lens was used to focus the laser beams produced by a dual Nd-YAG laser into the fluid. A cylindrical lens was then used to expand the laser beam into a sheet with a thickness of about $1 \mathrm{~mm}$.

For the velocity field measurements in the horizontal plane, the laser sheet was introduced horizontally about $2 \mathrm{~mm}$ below the water surface with the camera pointed upwards, as shown in figure 1, to have an un-obscured view of the flow field. For the velocity field measurements in the vertical plane, the laser sheet was introduced vertically from the bottom of the container along its axis of symmetry with the camera mounted almost horizontally.

Fluorescent beads with diameter less than $30 \mu \mathrm{m}$ were added to the water to facilitate the PIV measurements. They absorb the incoming illumination at $532 \mathrm{~nm}$ and re-emit light at around $580 \mathrm{~nm}$. A CCD camera with a $28 \mathrm{~mm}$ lens was used to record images with dual-flash illumination at a frequency of $10 \mathrm{~Hz}$. Sizeable particle displacements corresponding to various velocities were achieved by varying the pulse interval from 4.5 to $25 \mathrm{~ms}$. Stray light scattered off all the surfaces was eliminated by a $545 \mathrm{~nm}$ high-pass filter mounted on the camera that only allowed the fluorescent light from the fluorescent beads to pass. Possible image distortions were corrected after elaborate image calibrations. The velocity fields are obtained after processing 
(a)

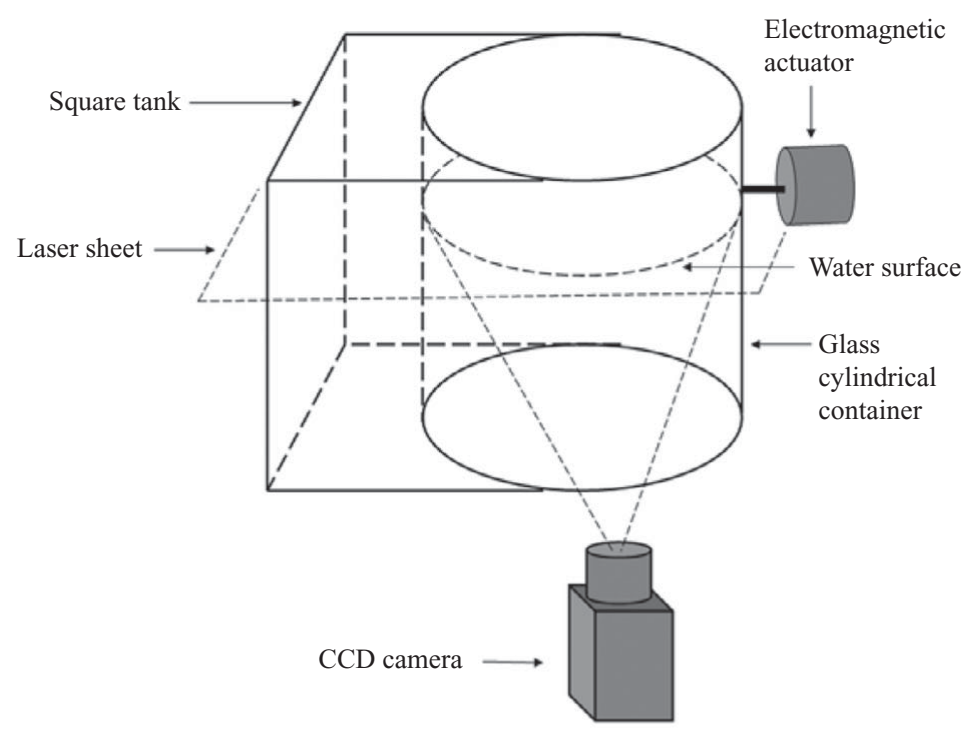

(b)

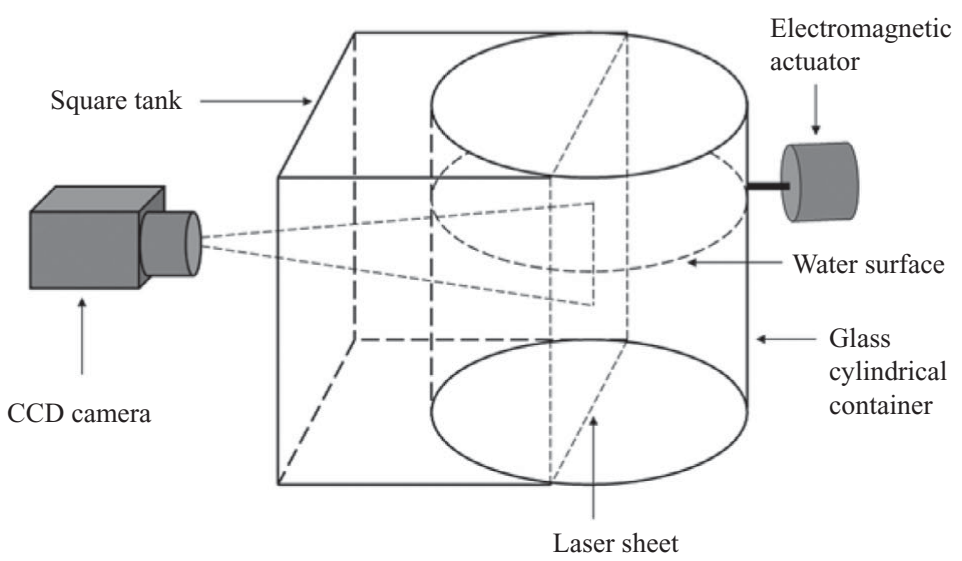

FIGURE 1. Schematics of the experimental setup together with the PIV arrangements. The cylindrical container was excited at the sidewall with an electromagnetic actuator with controlled amplitude and frequency. (a) For the velocity field measurements in the horizontal plane, the laser sheet was introduced horizontally through the front wall of a square container encasing one side of the cylindrical container. The CCD camera captured the images from below the container. (b) For the velocity measurements in the vertical plane, the laser sheet was introduced vertically into the fluid with the camera pointing slightly upwards $5^{\circ}$ below the fluid surface.

the dual-frame images using $32 \times 32$ pixel interrogation with $50 \%$ overlap based on the cross-correlation algorithm. Experiments were conducted with different water depths with robust phenomena observed repeatedly for all cases.

\section{Development of circular and azimuthal waves}

The circular capillary waves are hard to see with the naked eye due to their low amplitudes. Thus, the shadowgraph method is very useful to capture these waves. 


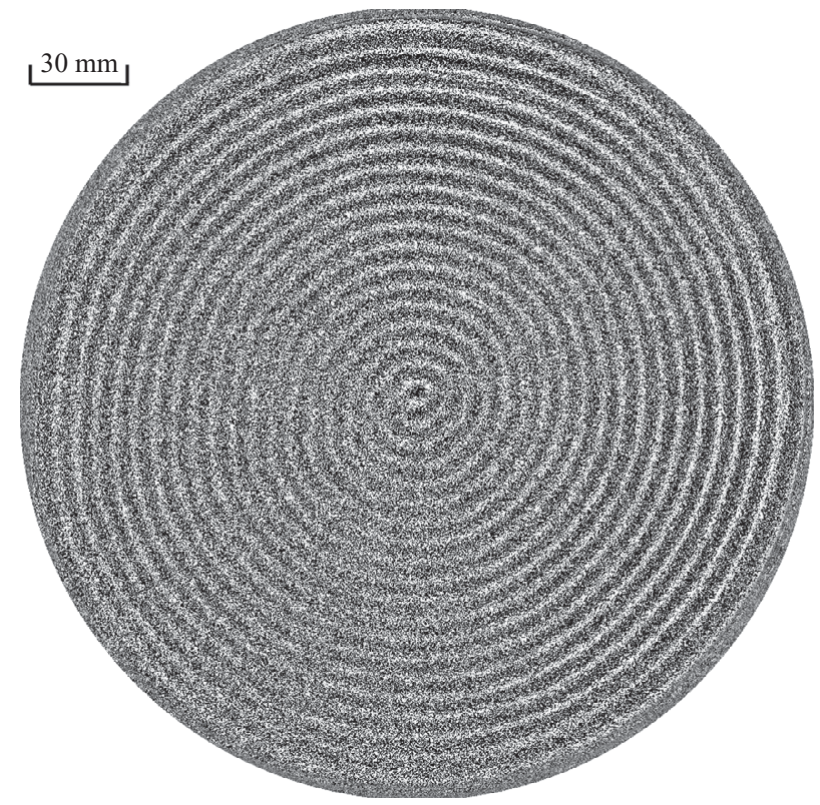

FIGURE 2. Photograph of the fluid surface at low-amplitude excitation. The waves are observed to propagate from the edge to the centre for an excitation frequency of $48.5 \mathrm{~Hz}$. The bright lines correspond to wave crests while the dark lines correspond to wave troughs.

The timing of the camera exposure, $t$, was fixed relative to the wave period, $T$, as $t=n T+k$. Here, $n$ is a positive integer and $k$ is much smaller than $T$. The interval between camera frames was about twice $T$. Fine tuning of the excitation frequency to introduce a slight mismatch of the two phases resulted in a movie showing the slow evolution of the surface waves.

The circular capillary waves have been shown to have the same frequency as the forcing frequency (Peng et al. 2005). The capillary waves were investigated here using a laser beam projected vertically towards the surface from below. For small excitation amplitudes, capillary waves with wavelengths corresponding to the excitation frequency appeared on the surface. These wave amplitudes were small but visible and could be easily detected by the laser beam refraction on the water surface, as shown in figure 2 . The dispersion of the capillary waves was also observed by Hsieh $(1994,1997,2000)$.

Increase of the excitation amplitude results in the appearance of azimuthal waves propagating in the anticlockwise circumferential direction. The frequency of the azimuthal waves is half of the excitation frequency (Peng et al. 2005, 2008).

When the azimuthal waves begin to emerge, the wave fronts are perpendicular to the container wall and propagate in the circumferential direction. The circular waves interacted with the azimuthal waves, forming quadrilateral shaped regions. Further increases of the forcing amplitude increased the amplitude of the azimuthal waves in the radial direction and their curvature. The azimuthal waves moved inwards with increasing length scale. Figure 3 shows the development of the azimuthal wave, with the wave amplitudes growing with increasing excitation wall acceleration. The red curves indicate the region where the azimuthal waves emerge. With increasing forcing amplitude, the influence regions on the surface grow to the centre of the basin and the wave-wave interactions between the surface waves intensify. As the external 
(a)
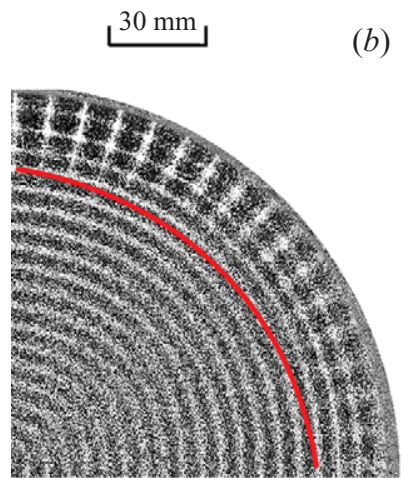

(c)

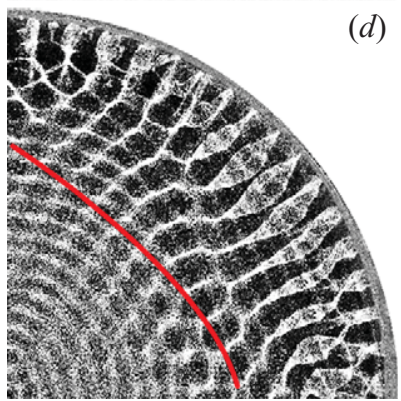

(b)
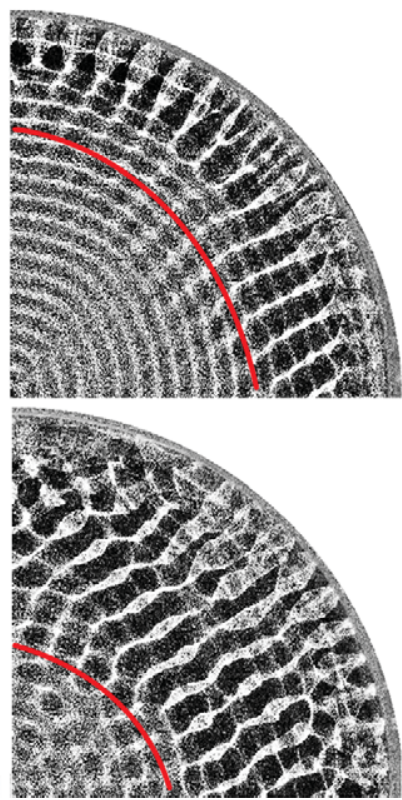

FIGURE 3. Evolution of azimuthal waves with increasing forcing amplitude. The wall acceleration (amplitude) was increased in this figure from 5.9 to $12.1 \mathrm{~m} \mathrm{~s}^{-2}(a)-(d)$ with an excitation frequency of $48.5 \mathrm{~Hz}$. The upper region of the red curve indicates where the azimuthal waves emerge. With increased forcing amplitude, the influenced regions grow inwards and the interactions between the circular waves and the azimuthal waves intensify.

forcing increases further, the azimutal waves gradually fill the whole circumference and become curved in the strongest vibrating regions.

\section{Wave motions}

One interesting phenomenon that often occurs in vibrated boundary layers is steady streaming (see the review by Riley (2001) for details). Rayleigh (1883) first studied the streaming phenomenon in a vibrated, velocity-dominated flow. He analysed the streaming formation mechanism induced by sound in a closed tube and found the Eulerian drift in the wall boundary layer. The steady streaming induced by a vibrated viscous fluid can be traced back to Faraday (1831). The streaming Reynolds number is defined as

$$
R e_{s}=\frac{U_{\infty}^{2}}{\omega \nu}
$$

where $v$ is the kinematic viscosity of the fluid and $U_{\infty}$ is the actual velocity of the outer flow due to the vibrations, which can be expressed as $A / \omega$ ( $A$ is the forcing acceleration and $\omega$ is the circular frequency). The flow can be classified according to the Reynolds numbers as the following.

(i) $R e_{s} \ll 1:$ The constant part of the outer flow in the Stokes layers is creeping flow, which is referred to as Rayleigh-Nyborg-Westervelt (RNW) streaming, as suggested by Lighthill (1978). 


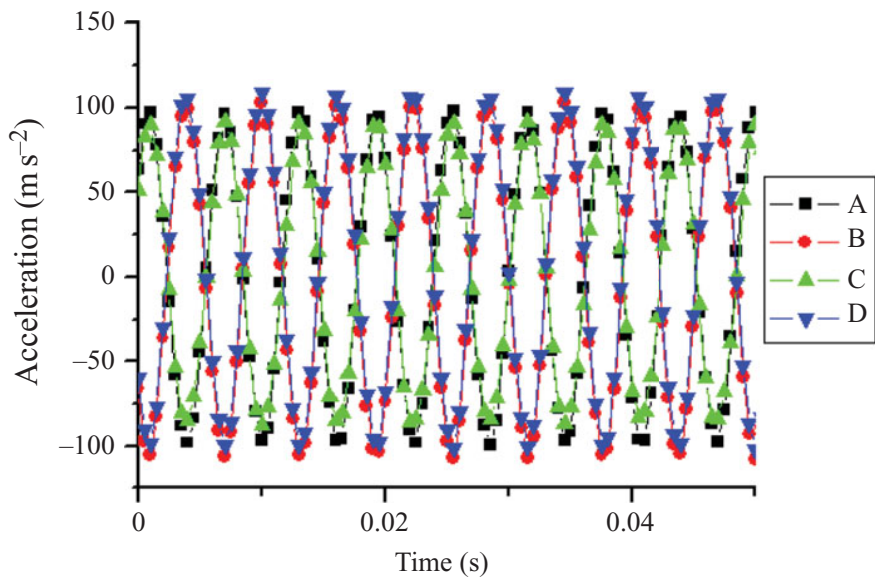

FIGURE 4. Phase relations for the accelerations at the four antinodes around the wall at a forcing frequency of $160 \mathrm{~Hz}$. A, B, C and D are the four antinodes around the wall shown in figure 5 .

(ii) $R e_{s} \sim 1$ : The constant part of the outer flow satisfies the full Navier-Stokes (N-S) equations and is called Stuart streaming (Lighthill 1978).

(iii) $R e_{s} \gg 1$ : The streaming layers become thin boundary layers, where streaming jets start to emerge. The streaming jet induced by the vibrated circular cylinder in the still fluid was first observed by Davidson \& Riley (1972) and has attracted many studies (Riley 2001, for example).

The wave phenomenon with $R e_{s} \sim 1$ is addressed in $\S 4.1$ and that with $R e_{s} \gg 1$ is addressed in $\S 5.1$.

\subsection{Streaming vortices for $R e_{s}<1$}

The circumferential waves initially develop at a forcing frequency of $160 \mathrm{~Hz}$ (circular frequency $\left.\omega=2 \pi f \sim\left(10^{3}\right) \mathrm{rad} \mathrm{s}^{-1}\right)$ and a forcing acceleration of $A=25 \sim O\left(10^{1}\right) \mathrm{m} \mathrm{s}^{-2}$. The characteristic velocity of the vibrating wall is $U_{\infty}=A / \omega \sim O\left(10^{-2}\right) \mathrm{m} \mathrm{s}^{-1}$ and the kinematic viscosity is $v=\left(10^{-6}\right) \mathrm{m}^{2} \mathrm{~s}^{-1}$, so the streaming Reynolds number is

$$
R e_{s}=\frac{U_{\infty}^{2}}{\omega \nu}=O\left(10^{-1}\right) .
$$

A time series of the wall accelerations at the four antinodes A, B, C and D are shown in figure 4 at $160 \mathrm{~Hz}$ excitation frequency. The wall oscillates at the same frequency as the actuation frequency of $160 \mathrm{~Hz}$. The data show that neighbouring points have opposite signs, i.e. their phase difference is $180^{\circ}$. Points that are not adjacent have the same sign and the same phase. Since the container wall vibrates with four antinodes (figure 4), the flow field on the free surface is divided into four roughly symmetric areas $(\mathrm{AEB}, \mathrm{BFC}, \mathrm{CGD}, \mathrm{DHA})$ as shown in figure 5 (top view). Antinodes A, B, C and D correspond to the four strongest vibrating points on the wall (antinodes) while nodes E, F, G and $\mathrm{H}$ correspond to the four weakest vibrating points on the wall. The initial development of the azimuthal waves can also be seen in figure 5. In the region near points $\mathrm{E}, \mathrm{F}, \mathrm{G}$ and $\mathrm{H}$, the fluid is translated towards the centre of the basin, while the fluid near points A, B, C and D is transmitted towards the wall. The physical mechanism responsible for the flow formation will be discussed later. 
(a)

A

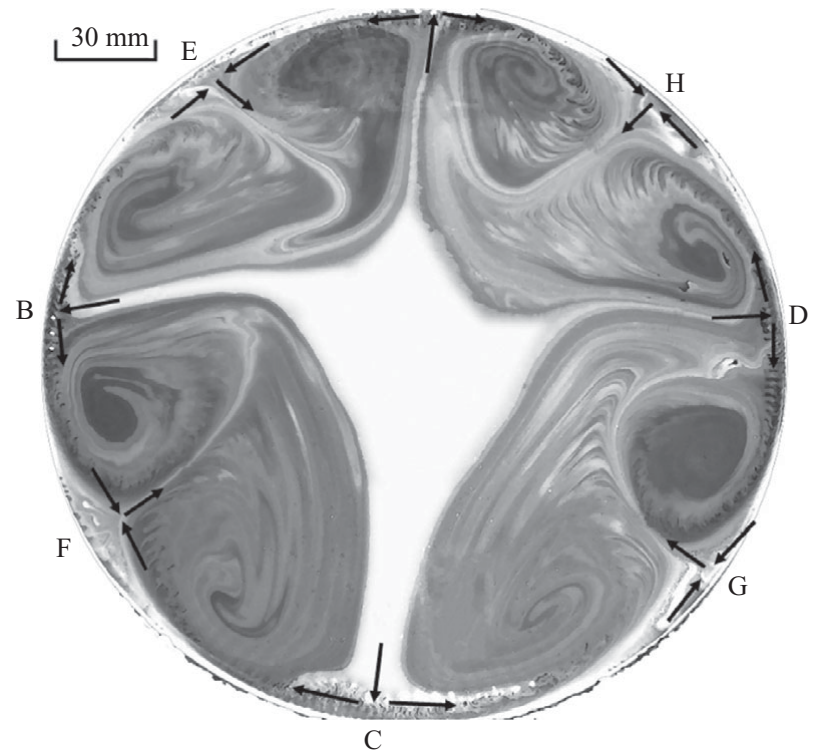

(b)

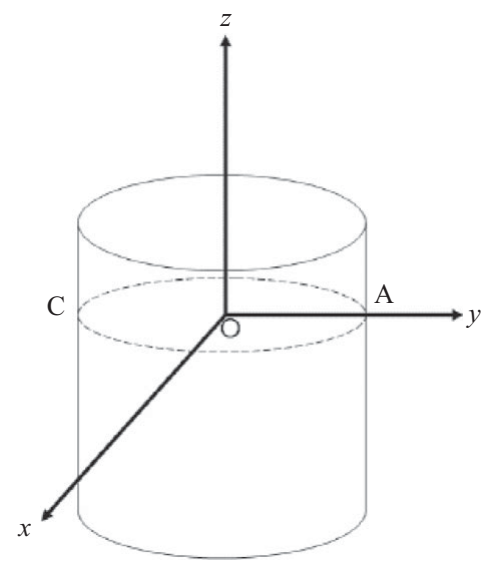

FIGURE 5. (a) Flow visualization as the azimuthal waves initially develop in the cylindrical container. This visualization was made possible by adding a small amount of ink along the wall-water contact line (the meniscus). The black arrows indicate the flow directions while points $\mathrm{A}, \mathrm{B}, \mathrm{C}$ and $\mathrm{D}$ correspond to the four strongest vibrating points on the wall (antinodes) and points $\mathrm{E}, \mathrm{F}, \mathrm{G}$ and $\mathrm{H}$ correspond to the four weakest vibrating points on the wall (nodes). The forcing point is at 12 o'clock near A. $(b)$ The Cartesian coordinate is defined with line $\mathrm{BD}$ as the $x$-direction, line CA as the $y$-direction and the $z$-direction being the vertical.

The corresponding velocity field, vorticity field and streamlines at this stage are shown in figure 6. The largest velocities in the horizontal plane were observed at the borders of each region (corresponding to $\mathrm{E}, \mathrm{F}, \mathrm{G}$ and $\mathrm{H}$ regions in figure 5). The four radial lines originating from the four nodes at the wall, with the fluid flowing inwards, converge at the centre to form a counterclockwise rotating square pattern. The second largest velocities lie on the boundaries between each clockwise rotating vortex and its neighbouring counterclockwise rotating vortex (corresponding to points $\mathrm{A}, \mathrm{B}, \mathrm{C}$ and $\mathrm{D}$ in figure 5). The four radial lines originating from the central rotating square pattern essentially follow the outward flow in the radial direction towards the four strongest vibrating points. The vorticity distribution has four diagonal lines forming four groups of vorticity sheets and a completely positive vorticity square with weaker vorticity levels between the four radial lines. The streamlines from the four diagonal lines form a counterclockwise spiral at the centre. These measurements agree well with the flow visualization shown in figure 5.

For measurements in the vertical plane aligned with points B and D (figure $6 b$ ), the strongest flows are in the radial directions injected from the surface (points B and D) with high vorticity in the thin shear layer near the surface. The streamlines show two recirculating regions.

\subsection{Water droplet ejection}

Further increases of the forcing amplitude lead to droplet ejection which starts from the four strongest vibrating points. The droplet ejections are fast with high ejection 

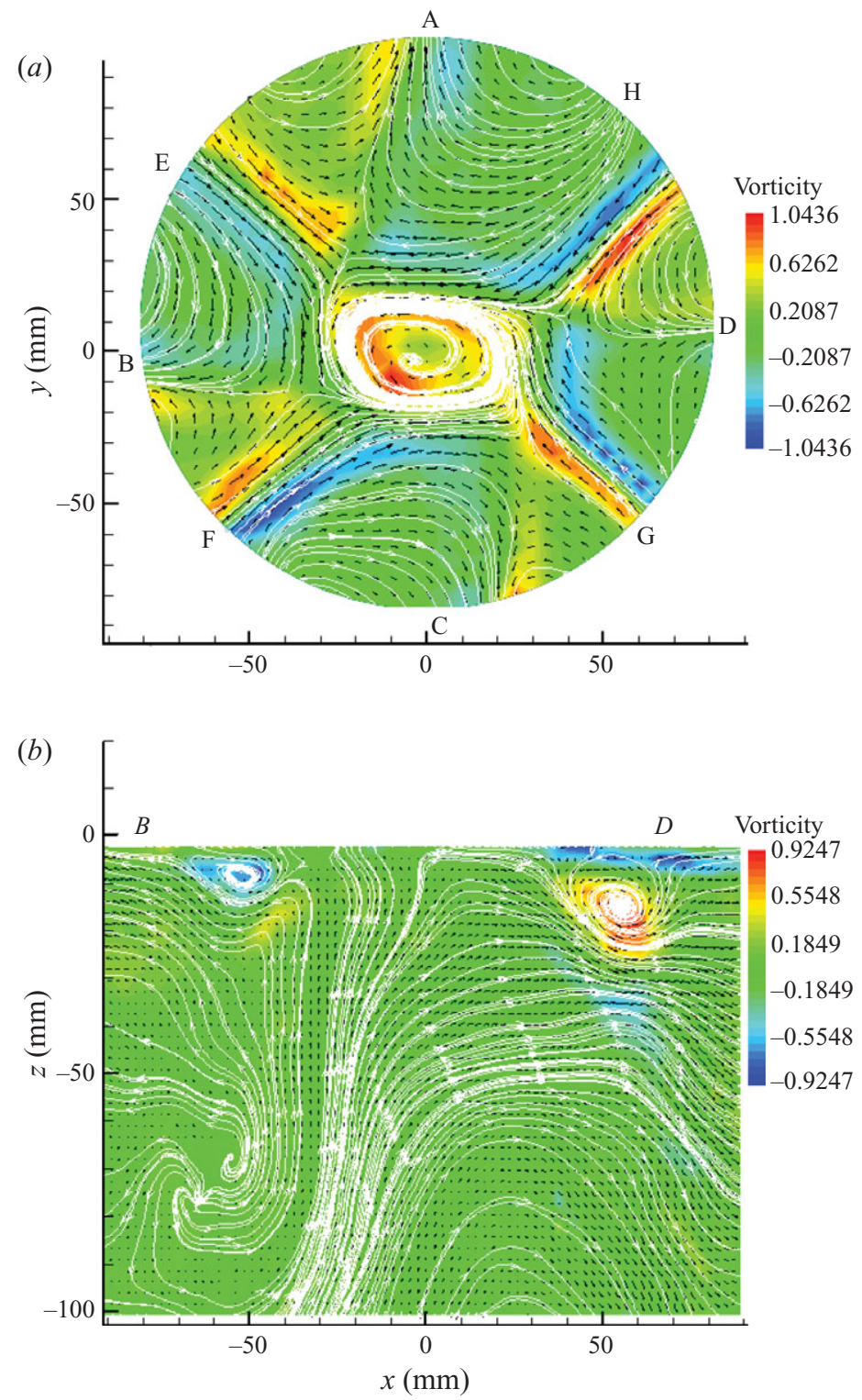

FIGURE 6. Velocity vectors (dark arrows), vorticity contours (filled colour) and stream lines (white curves) as the circumferential waves initially develop in the glass container for a forcing frequency of $160 \mathrm{~Hz}$ and a forcing acceleration of $25 \mathrm{~m} \mathrm{~s}^{-2}$. Points A, B, C and D correspond to the four strongest vibrating points on the wall (antinodes) and points $\mathrm{E}, \mathrm{F}, \mathrm{G}$ and $\mathrm{H}$ correspond to the four weakest vibrating points on the wall (nodes). (a) Velocity and vorticity measured in a horizontal plane, $(b)$ velocity and vorticity measured in a vertical plane aligned with points $\mathrm{A}$ and $\mathrm{C}$.

velocities. The wave crests are elongated with the circular drops forming on the top. The spherical droplets are rapidly ejected from the wave crests due to the strong surface tension effect. The flow structures formed when forcing frequencies are near the resonance frequency and the classic edge condition at the contact line described by the weakly nonlinear theory developed by Sun et al. (1995) which did not include 


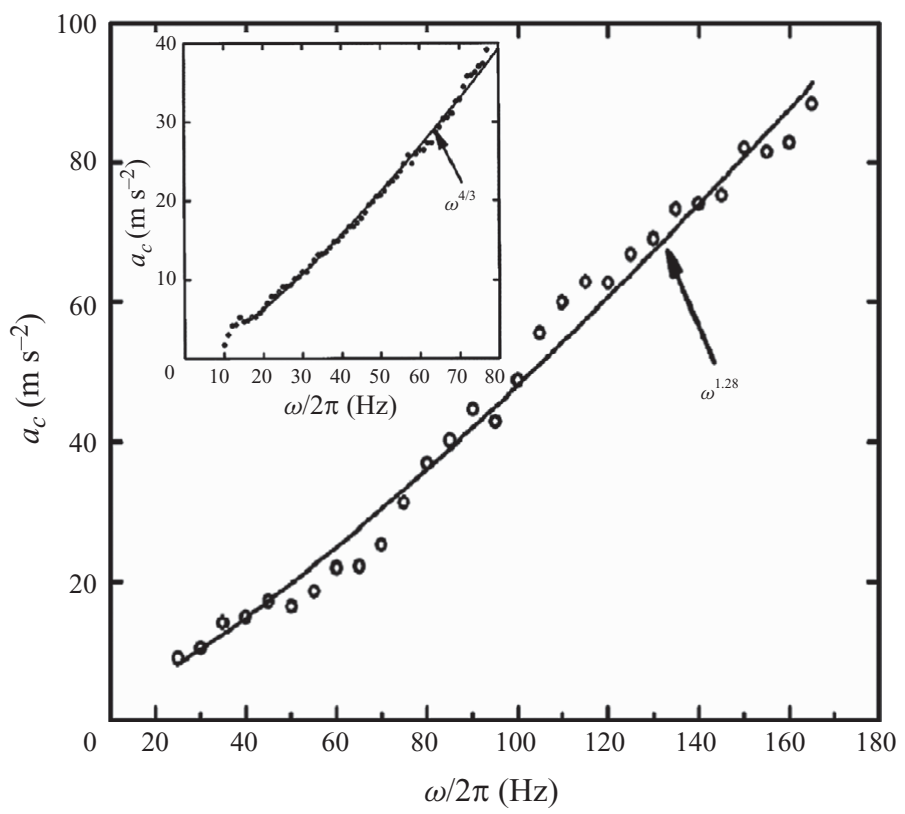

FIGURE 7. Critical acceleration for droplet ejection in water in a circular Plexiglas container with $a_{c}=0.13226(\omega / 2 \pi)^{1.28}$ for frequencies in the capillary range. The inset shows the results of Goodridge, Shi \& Lathrop (1996).

the surface tension effect. A two-step asymptotic expansion in time was developed by Chang \& Shen (2000b). Their amplitude equations show that the amplitudes of the excited waves increase with time until surface wave singularities are produced to form droplets, spray and foam. When the water droplets jump and splash on the surface, the organized flow patterns are no longer evident. Wei et al. (1997) observed strong surface flow towards the centre in this state. The minimum wall velocities necessary for droplets to leave the surface were measured for various frequencies by Hsieh \& Denissenko (1998).

The waves that produce droplets were characterized by the measured threshold accelerations as a function of the forcing frequency in the container at a water depth of $160.0 \mathrm{~mm}$. Long-lasting transient states were observed throughout the measured frequency range. The thresholds were determined by supplying sufficient forcing to produce vigorous droplet ejection and then lowering the magnitude until the ejections stopped. The droplets were detected visually after a sufficient time interval to make sure that all transient states had passed. The threshold accelerations for droplet ejection for frequencies from 25 to $165 \mathrm{~Hz}$ are shown in figure 7 . The critical acceleration is a function of the frequency of the lateral excitation for a given surface tension and fluid viscosity. A least-squares fit of this acceleration data for water in the capillary range was similar to that of Goodridge, Shi \& Lathrop (1996), as shown in the inset of figure 7. This confirms the dependence of the threshold acceleration for droplet ejection on the forcing frequency. When the droplet splashing started, the radial velocity at the four antinodes A, B, C and D was observed to reverse direction. This reversal corresponds to the two kinds of steady streaming phenomena for different Reynolds numbers discussed in $\S 5.1$. 


\section{Low-frequency, three-dimensional gravity waves and droplet splashing mechanism}

\subsection{Four streaming jets for $R e_{s} \gg 1$}

Jet-like flows do not form at low forcing amplitudes until the increasing amplitude of the oscillating wall begins to generate local jet-like flows ejected into the interior fluid, which is similar to the streaming phenomena produced by a loudspeaker.

For the case of a streaming jet, as reported by Davidson \& Riley (1972), the jet-like flow arises when the streaming Reynolds number is large. Droplet ejection begins when the streaming velocity is $U_{\infty}=O\left(10^{0}\right) \mathrm{m} \mathrm{s}^{-1}$ and the streaming Reynolds number is $R e_{s}=O\left(10^{3}\right) \gg 1$.

When the local jets from opposite sides are strong enough to interact, they both turn downwards and eventually form four global streaming jets with four large global vortices as shown in figure 8 .

This was studied in more details by measuring the flow pattern with a PIV system with the fluid seeded with fluorescent beads. These measurements provided instantaneous velocity and vorticity fields. As illustrated in figure 8, the measurements in the horizontal plane show that the largest inward velocities originate from the four strongest vibrating points at the wall. The jets follow four radial lines from points A, B, C and D towards the centre. These four jet-like flows cause strong shear layers that create most of the vorticity. The vorticity fields become regular and disperse at the free surface with the generation of many small vortices as the waves break. The vortex generation mechanism at a free surface was described in $\mathrm{Wu}, \mathrm{Ma} \& \mathrm{Zhou}$ (2006).

The measurements in the vertical plane show several recirculating regions with strong surface flows towards the centre. The shedding vortices move along a curved path, inwards and downwards, leading to large opposing recirculation regions. As a result, the vorticities continually diffuse from the surface to the interior. The streamlines describe two large recirculation regions from the two sidewalls to the centre bottom, which can be regarded as a pair of steady streaming vortices.

Figure $9(a)$ shows the velocity distributions along the streaming-jet direction at five positions along the $Y$-axis at intervals of $3.4 \mathrm{~mm}$. Here, $X$ measures the distance from the centre of the cylinder and $Y$ is the distance from the wall. The results show that the velocity in the $y$ distribution $V$ is similar to that of a jet, i.e. the largest velocity is at the centre and the smallest velocities are at both sides. All the velocities then decrease with increasing distance from the wall. Figure $9(b)$ shows horizontal cross-flow distributions of $U$ at the same five positions. The centre velocity is nearly zero owing to the flow field symmetry. The absolute values of $U$ also decrease with distance from the wall, which also agrees with normal jet flow. However, the jets in the present situation are not the same as isolated jets since they interact strongly with neighbouring jets in narrow regions near the centre, which causes the horizontal velocities at the two sides to first decrease to zero, then change direction and increase. The overall velocity distribution is somewhat like a rhombus.

\subsection{Jet instabilities}

The four jets were found to oscillate symmetrically as shown in figure 10, where the curves represent the simultaneous measurements of the vertical velocity at four symmetrical points close to the four strongest vibrating locations A, B, C and D. The vortex originating from the boundary layer (Lee \& Wu 2008) that is shed around the 

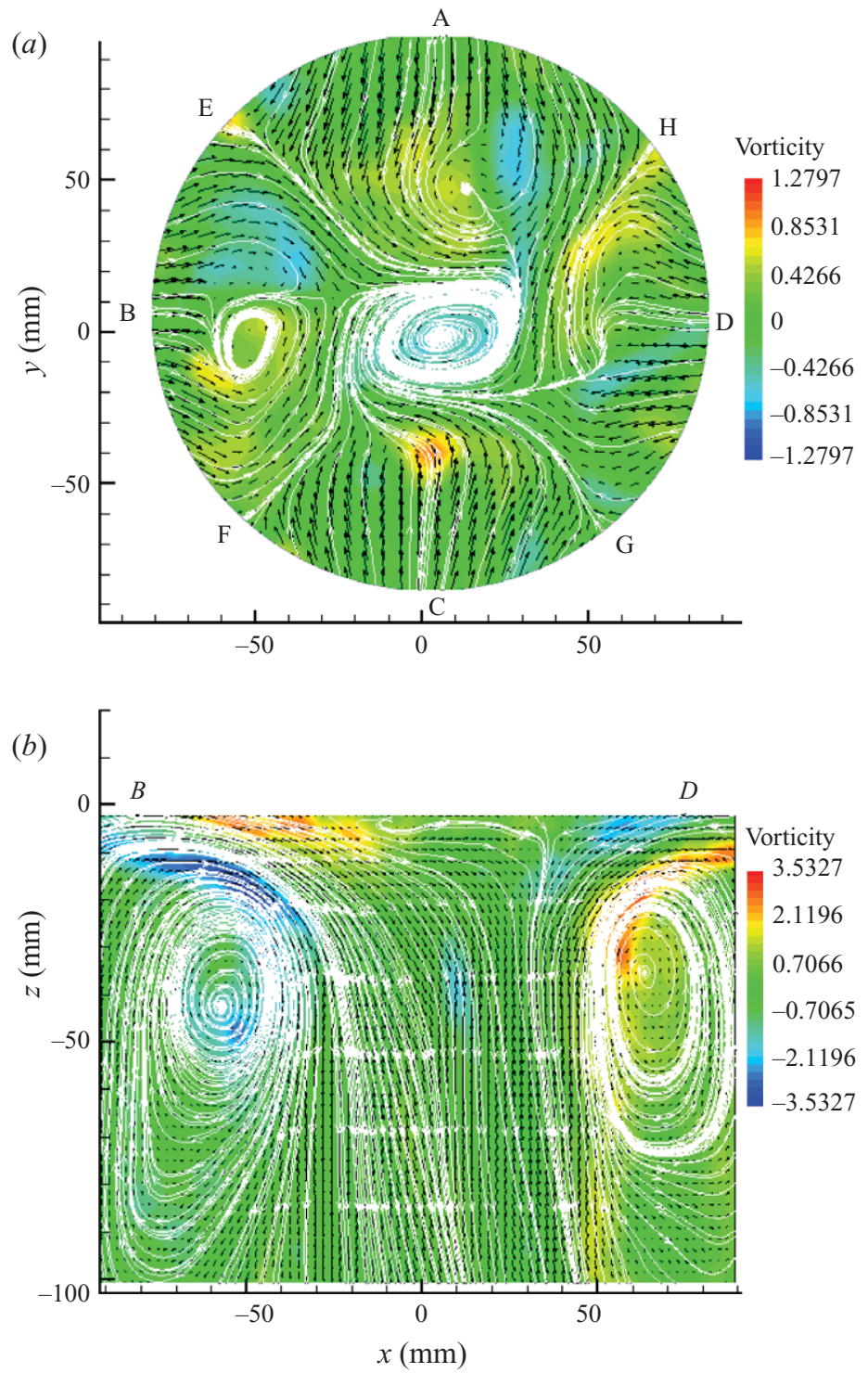

FIGURE 8. Mean velocity vectors (dark arrows), vorticity contours (filled colour) and streamlines (white curves) inside the container during the droplet ejection regime at a forcing frequency of $160 \mathrm{~Hz}$ and a forcing acceleration of $120 \mathrm{~m} \mathrm{~s}^{-2}$. Points A, B, C and D where the four jet-like flows emerge, correspond to the four strongest vibrating points on the wall (antinodes). (a) Velocity and vorticity fields measured in a horizontal plane $2 \mathrm{~mm}$ below the surface. $(b)$ Velocity and vorticity fields measured in the vertical plane aligned with points B and D.

jets was studied to clarify this phenomenon. Figure 11 illustrates a series of vortex rings shed around the jets due to the Kelvin-Helmholtz instability. The period of the vortex shedding was estimated to be $0.3 \mathrm{~s}$. Ho \& Huerre (1984) reviewed linear instability theory and experimental investigations in mixing layers and pointed out that for all laminar mixing layers the most amplified waves collapse at a single 

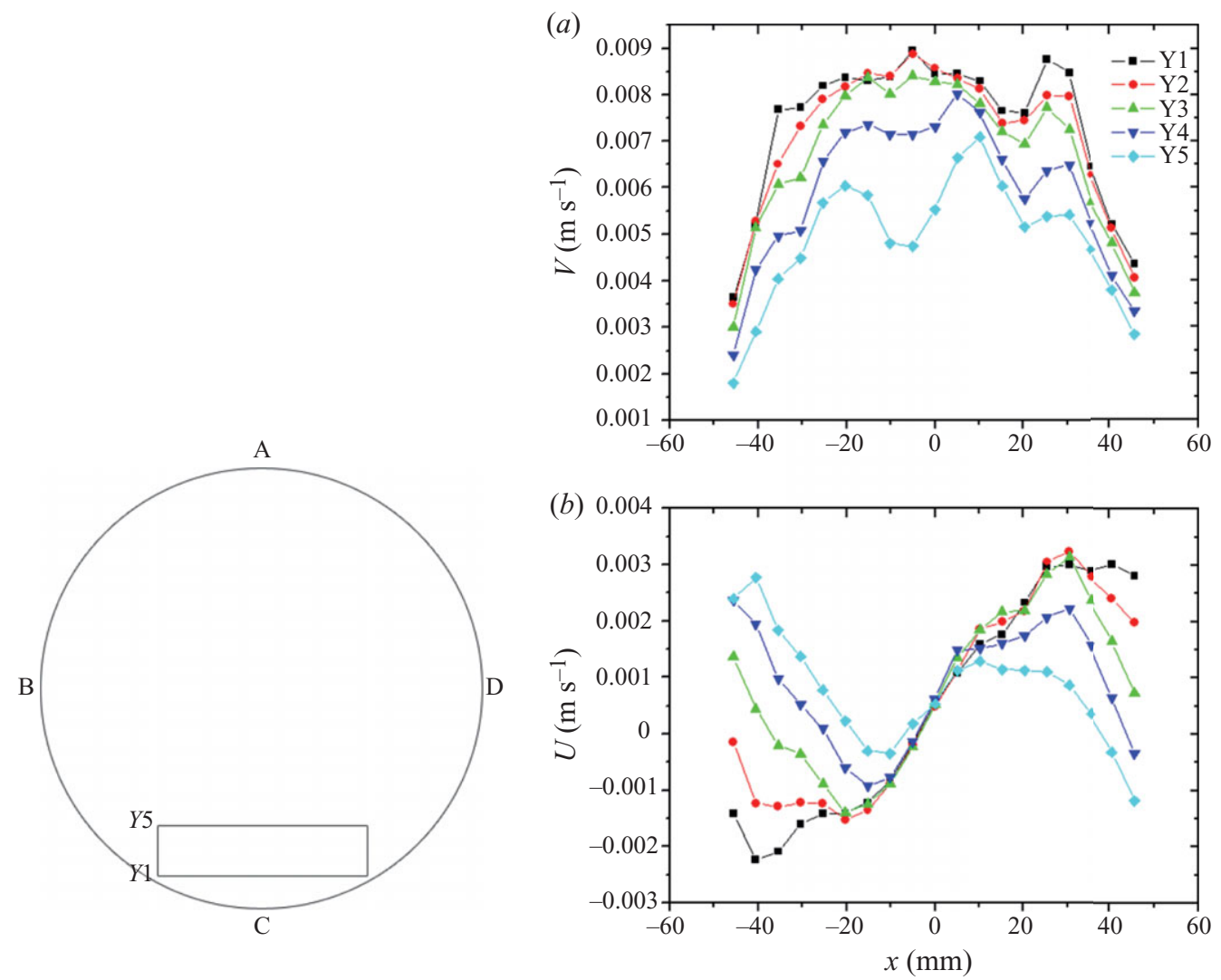

FIGURE 9. Jet profiles and velocity distributions in the streaming and cross-flow directions at a forcing frequency of $160 \mathrm{~Hz}$ and a forcing acceleration of $120 \mathrm{~m} \mathrm{~s}^{-2}$. The flow conditions are the same as in figure 8. (a) Velocity distributions in the streaming direction. (b) Velocity distributions in the cross-flow direction. $X$ is the distance from the centre of the cylinder and $Y$ is the distance from the wall. $U$ is along the $X$ direction and $V$ is along the $Y$ direction.

Strouhal number 0.032 with an error of only $5 \%$.

$$
S t=\frac{f \theta}{\bar{U}}=0.032,
$$

where $f$ is the most unstable frequency, $\theta$ is the momentum larger thickness and $\bar{U}=\left(U_{1}-U_{2}\right) / 2$, where $U_{1}=U_{s}$ and $U_{2}=0$. For the present case, the streaming velocity of the induced jets is

$$
U_{s} \sim-\frac{3}{4 \omega} U_{0} \frac{\mathrm{d} U_{0}}{\mathrm{~d} x} \sim \frac{U_{0}^{2}}{\omega x}=\frac{A^{2}}{\omega^{3} x},
$$

where $\omega=160 \times 2 \pi=1005 \mathrm{rads}^{-1}$ is the circular frequency, $x$ is the thickness of oscillation boundary layer and $A=160 \mathrm{~m} \mathrm{~s}^{-2}$ is the forcing acceleration at the wall.

The streaming velocity measured by the PIV scheme at the threshold velocity needed to induce the free surface up-down motion was measured to be $U_{s}=0.0237 \mathrm{~m} \mathrm{~s}^{-1}$ (see figure $16 c$ ) at $x=1.05 \mathrm{~mm}$, where $\bar{U}=U_{s} / 2$,

$$
\theta=\int_{0}^{\infty} \frac{u}{U}\left(1-\frac{u}{U}\right) \mathrm{d} y=0.664 \frac{x}{\sqrt{R e}}=0.133 x=0.140 \mathrm{~mm}
$$




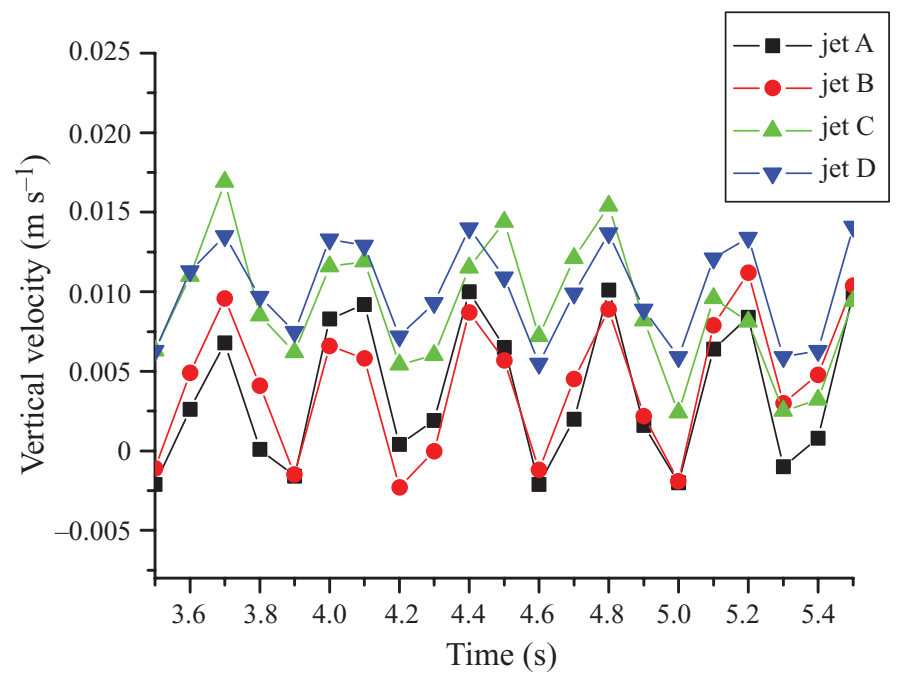

FIGURE 10. Jet oscillations at a forcing frequency of $160 \mathrm{~Hz}$ and a forcing acceleration of $120 \mathrm{~m} \mathrm{~s}^{-2}$. The data show simultaneous measurements of the vertical velocities at four symmetrical points close to the four strongest vibrating locations $\mathrm{A}, \mathrm{B}, \mathrm{C}$ and $\mathrm{D}$.

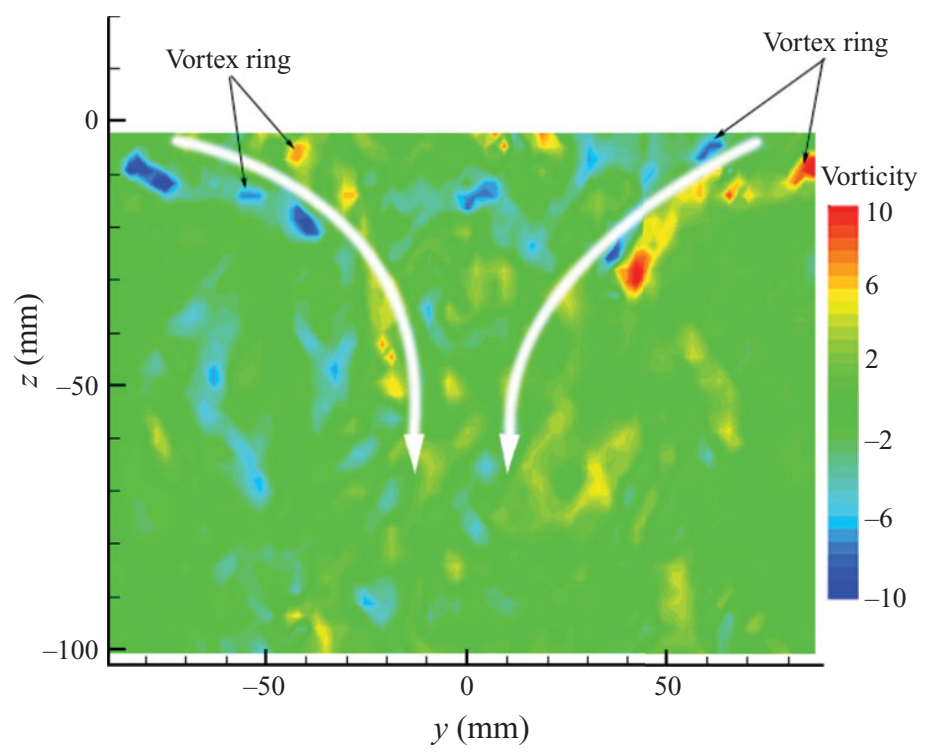

FIGURE 11. Series of vortex rings shed around the jets (shown by the white arrows) due to the Kelvin-Helmholtz instability. The forcing frequency is $160 \mathrm{~Hz}$ and the forcing acceleration is $120 \mathrm{~m} \mathrm{~s}^{-2}$.

where

$$
R e=\frac{U_{s} x}{v} .
$$

According to the definition of the Strouhal number, the most unstable frequency for the jets is $2.70 \mathrm{~Hz}$, which is close to our current estimation of $2.80 \mathrm{~Hz}$. Thus, the vortex shedding in this limited space is likely due to direct feedback from the vortex shedding from the global jets that causes the global jets to oscillate. 

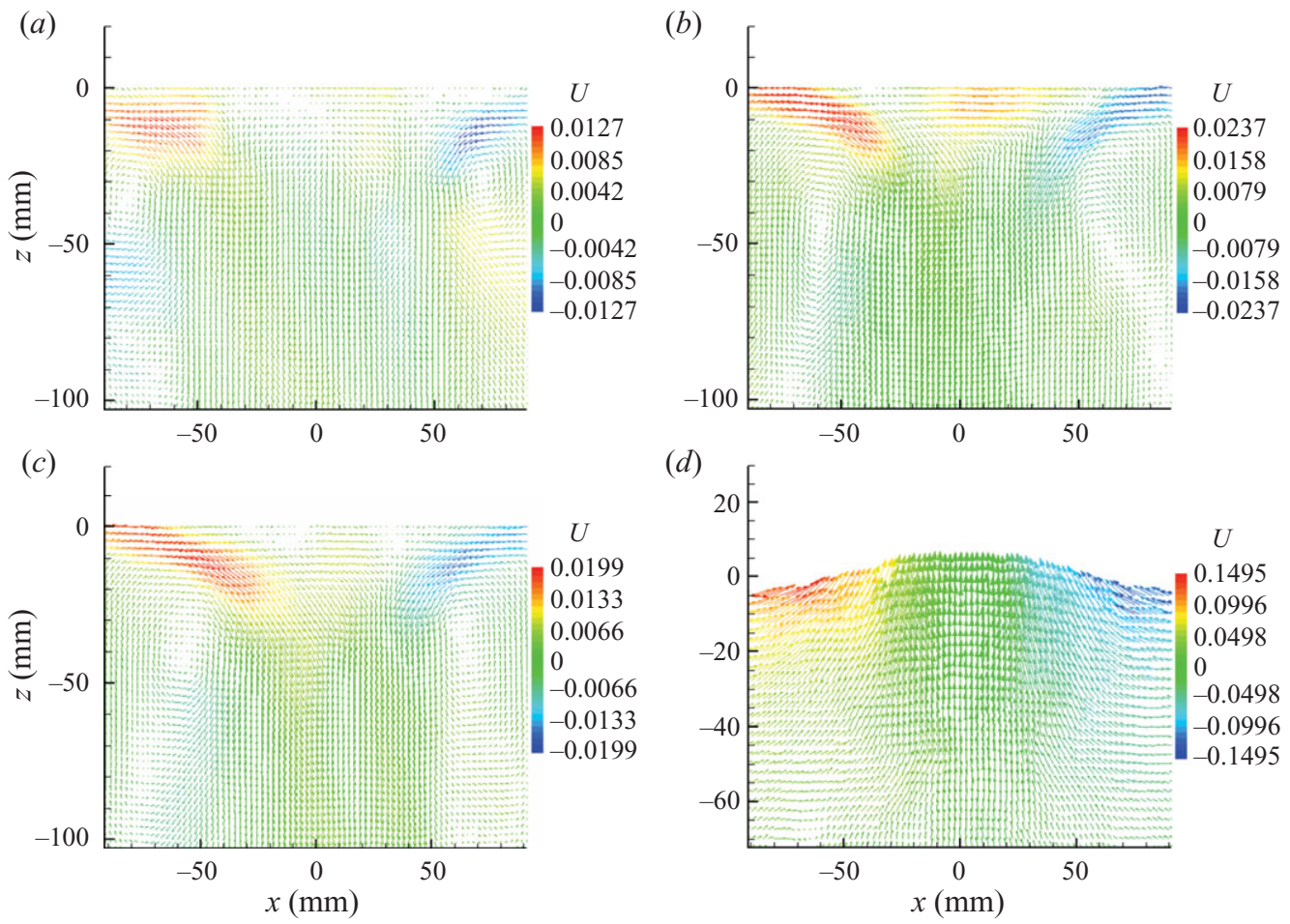

FIGURE 12. Interactions between the jets and the free surface at a forcing frequency of $160 \mathrm{~Hz}$. (a) Initially weak streaming jets for a forcing acceleration of $106.0 \mathrm{~m} \mathrm{~s}^{-2}$. (b) Stronger streaming jets for a forcing acceleration of $120 \mathrm{~m} \mathrm{~s}^{-2}$. (c) Streaming jets intensively interacting with the free surface for a forcing acceleration of $145 \mathrm{~m} \mathrm{~s}^{-2}$. (d) Low-frequency three-dimensional gravity waves emerge with a forcing acceleration of $170 \mathrm{~m} \mathrm{~s}^{-2}$.

\subsection{Generation of free surface waves by streaming jets}

One of the most impressive surface phenomena is the oscillation-induced jets and jet-induced sloshing by lateral single point forcing on the sidewall of the water-filled container. Saeki et al. (2001) observed self-induced free-surface oscillation termed 'self-induced sloshing' in a rectangular tank with a submerged and horizontally injected water jet. Self-induced sloshing is excited by the flow itself without any external forcing. This behaviour was also observed in the present experiments. The dominant frequency was found to be close to the first or second eigenvalue of the fluid in the tank. Four submerged water jets injected horizontally from the sidewalls propagated simultaneously towards the centre of the basin. As shown in figure 11, these four jets oscillated symmetrically. To satisfy fluid continuity, the fluid surface has to response accordingly. Figure 12 illustrates the interactions between the jets and the free surface. In figure 12(a) the streaming jets are instantly weak at a forcing acceleration of $106.0 \mathrm{~m} \mathrm{~s}^{-2}$. The dark red and blue regions correspond to the left and right jets originating from the oscillatory wall boundary layer. The jets extend only short distances from the wall and dissipate due to viscous effects with a low initial velocity of $1.26 \mathrm{~cm} \mathrm{~s}^{-1}$. As shown in figure $12(b)$, the streaming jets grow stronger with a forcing acceleration of $120 \mathrm{~m} \mathrm{~s}^{-2}$ and a maximum velocity of $1.98 \mathrm{~cm} \mathrm{~s}^{-1}$. A large circulation flow develops that transports the wall vorticity from the edge to the centre. In figure 12(c) the streaming jets interact intensively with the free surface at a forcing acceleration of $145 \mathrm{~m} \mathrm{~s}^{-2}$ with the initial jet velocity reaching a maximum of 


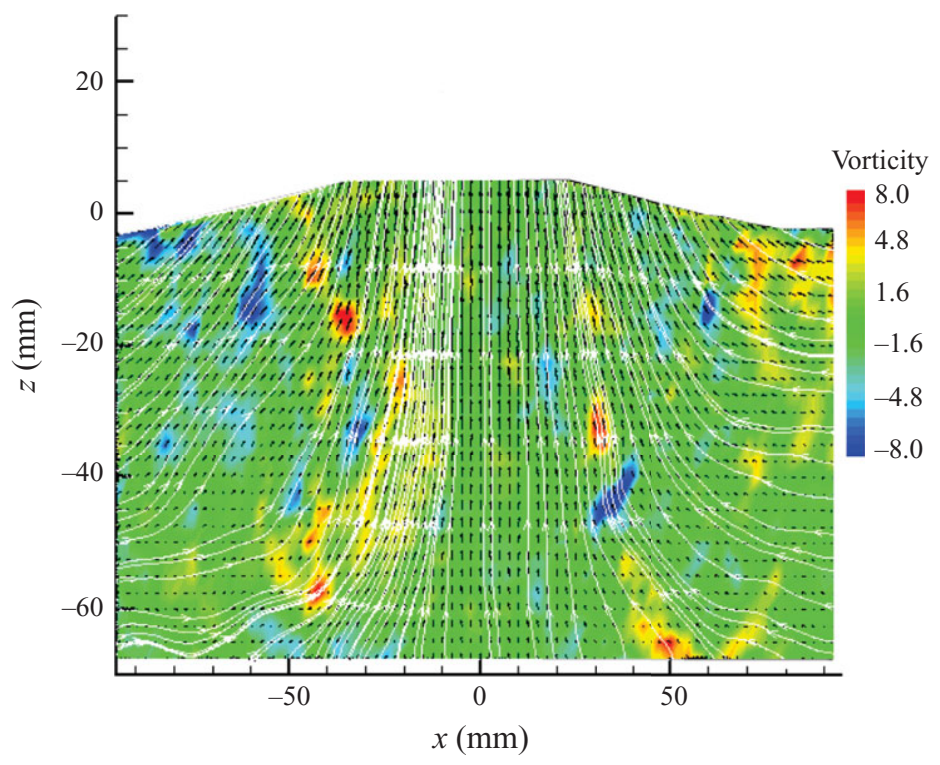

FIGURE 13. PIV measurements of a three-dimensional gravity wave in the container during the rising stage at a forcing frequency of $160 \mathrm{~Hz}$ and a forcing acceleration of $170 \mathrm{~m} \mathrm{~s}^{-2}$. The dark arrows represent the velocity vectors, the colour contours represent the vorticity field for the $(0,1)$ mode low-frequency three-dimensional gravity wave, and the white curved arrows represent the streamlines.

$2.37 \mathrm{~cm} \mathrm{~s}^{-1}$. The free surface begins to oscillate up and down with small amounts of energy gradually transferred to the free surface from the unsteady jets. In figure $12(d)$ low-frequency three-dimensional gravity waves emerge at a forcing acceleration of $170 \mathrm{~m} \mathrm{~s}^{-2}$. The maximum jet velocity increases to $14.9 \mathrm{~cm} \mathrm{~s}^{-1}$ and the jets coalesce with the three-dimensional gravity waves. The jet locations are now hard to distinguish since the fluid velocities in the container oscillate as a whole and the free surface position changes constantly.

Figure 13 shows the PIV results for the $(0,1)$ mode three-dimensional gravity wave at the rising stage. The fluid, on the two sides, moves inwards and pushes the middle part up. The centre points on the free surface have the largest velocities, with magnitudes of $20 \mathrm{~cm} \mathrm{~s}^{-1}$, about 10 times that of the induced jet velocities. This results in intermittent droplet ejection.

The vortices are mostly created in the boundary layers of the free surface (Wu et al. 2006) and along the wall and are transferred to the interior by the large vertical movements of the $(0,1)$ mode standing three-dimensional gravity wave. Once the $(0,1)$ mode three-dimensional gravity waves emerge, the up-down motion can persist for a long time as long as the external forcing conditions remain. The standing threedimensional gravity wave has a large inertia, and the forcing amplitude can be reduced by $50 \%$ without disturbing the vertical motion of the three-dimensional gravity wave. Therefore, there is a hysteresis effect between the critical forcing amplitudes needed to excite the three-dimensional gravity waves and the much lower values of the forcing amplitudes leading to extinction of the three-dimensional gravity waves. The interactions between the jets and the free surfaces are clearly illustrated by the timeseries variations of the jet and free surface velocities at the selected points shown in figures $14(a)$ and $14(b)$ for various forcing accelerations. The jet velocity is measured 

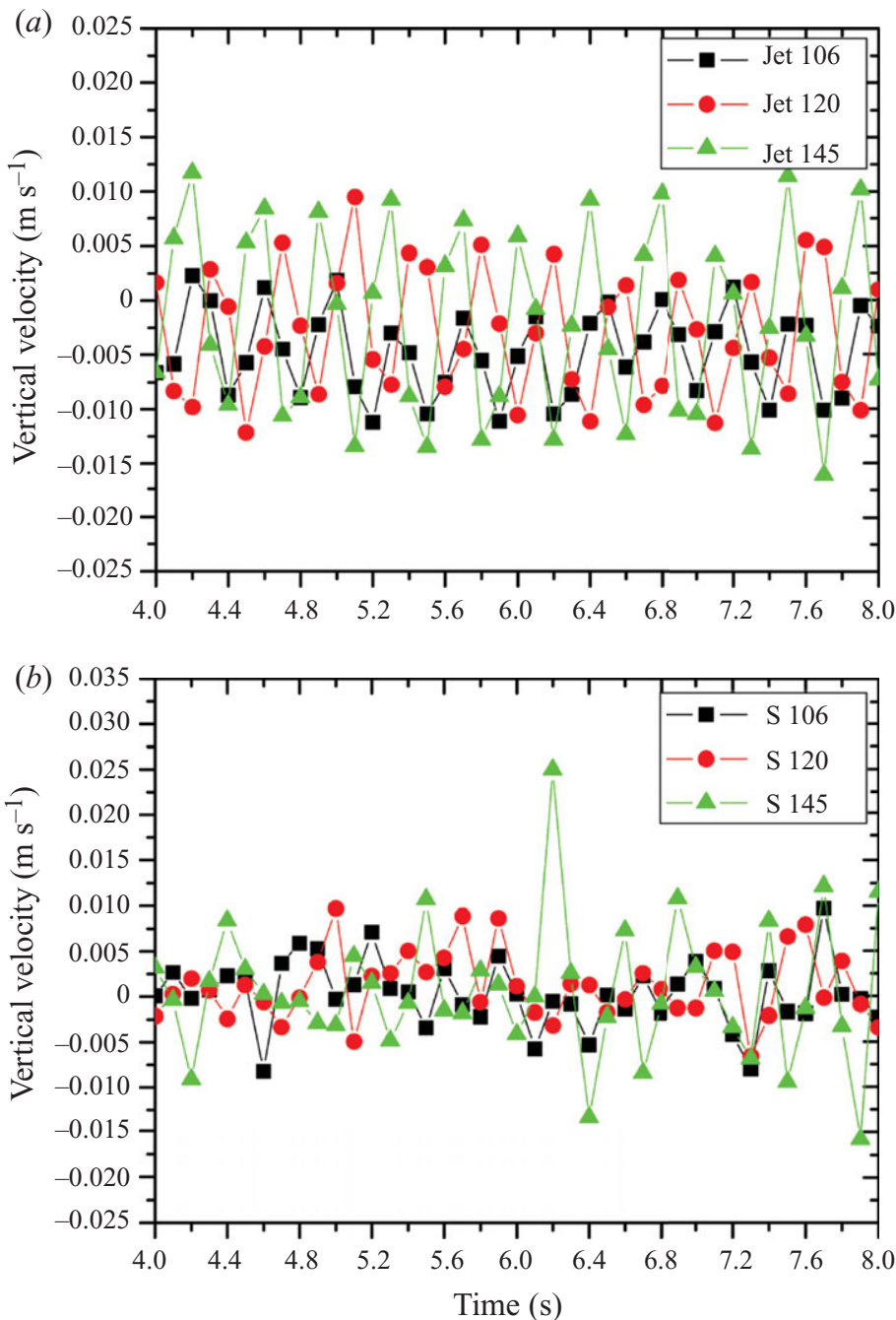

FIGURE 14. Jet and free surface velocities with increasing forcing accelerations for a forcing frequency of $160 \mathrm{~Hz}$. (a) Jet velocities at three forcing accelerations of 106,120 and $145 \mathrm{~m} \mathrm{~s}^{-2}$. (b) Free surface velocities at three forcing accelerations of 106,120 and $145 \mathrm{~m} \mathrm{~s}^{-2}$. The jet velocities were measured at point B in figure $8(b)$ while the free surface velocities were measured at the centre of the free surface.

at point $\mathrm{B}$ in figure $8(b)$ while the free surface velocity is measured at the centre of the free surface. The characteristic velocities of the jet and the free surface both increase with increasing forcing acceleration. The jet velocities are greater than the free surface velocities at the beginning, but the free surface velocities then increase faster than the jet velocities. Therefore, the maximum velocity on the free surface eventually exceeds the jet velocity, which indicates the significant energy transfer from the jets to the free surface.

\subsection{Low-frequency gravity waves and the feedback mechanism}

Although the dominant mechanism for droplet ejection is not the resonance between the external excitation frequency and the fluid behaviour, resonance does occur 

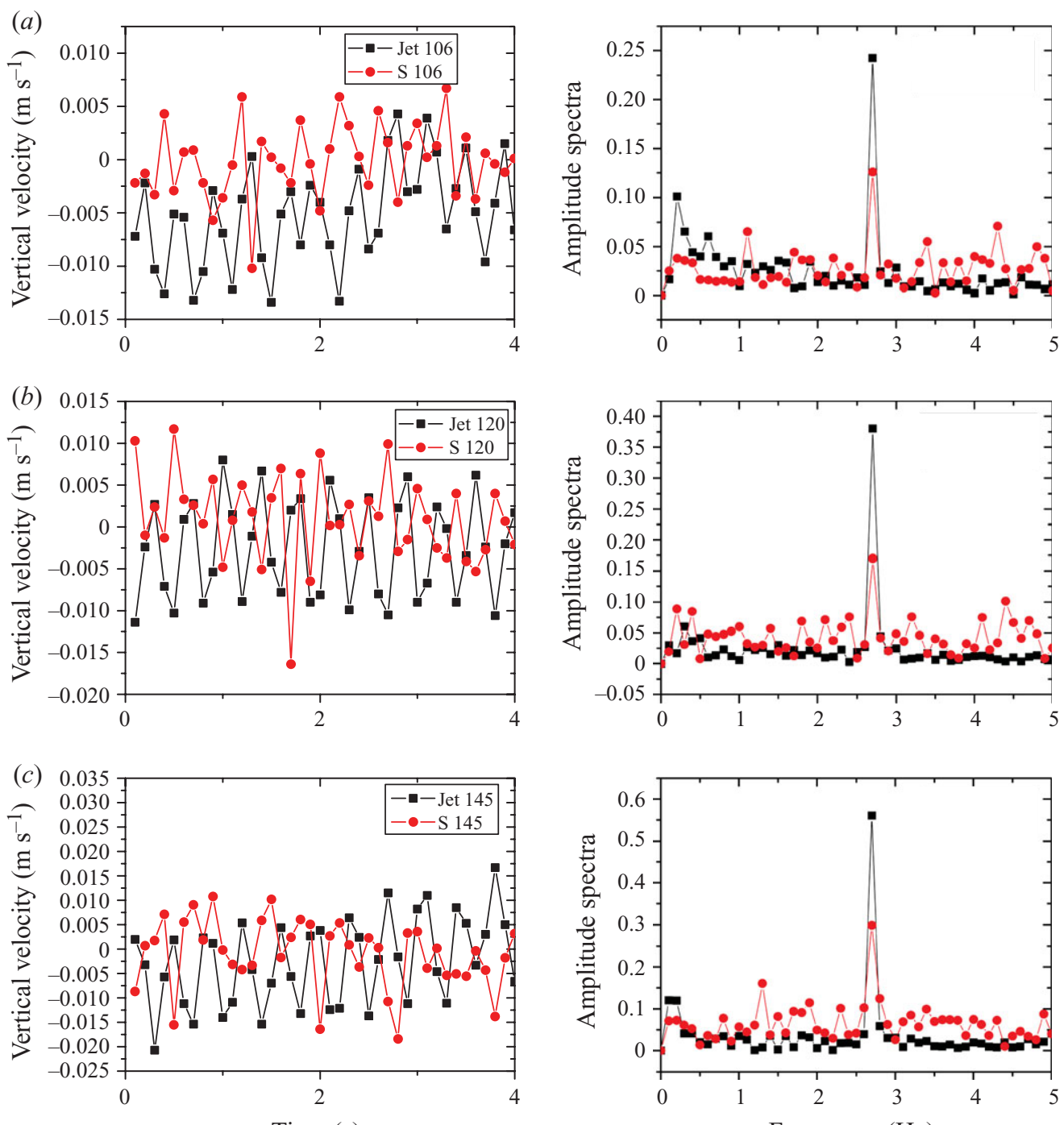

Time (s)

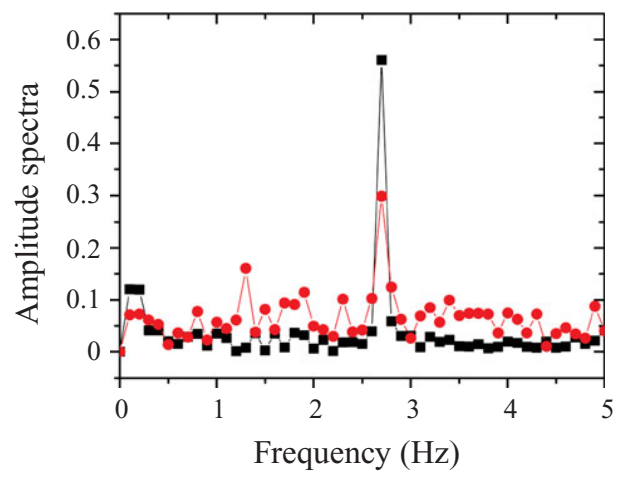

FIGURE 15. Phase and spectral relations between the jet and the free surface at a forcing frequency of $160 \mathrm{~Hz}$. $(a),(b)$ and $(c)$ correspond to forcing accelerations of 106, 120 and $145 \mathrm{~m} \mathrm{~s}^{-2}$, respectively.

between the vortex shedding, vortex oscillations and the surface waves. Some resonance modes create large amplitude standing three-dimensional gravity waves when the excitation amplitude is increased further after the appearance of the jumping droplets. The high-frequency capillary waves and the jumping droplets are apparent at high excitation frequencies but are suppressed during the three-dimensional gravity wave phase when the water level is higher near the wall than in the centre. The surface waves evolve towards low-frequency three-dimensional gravity waves and eventually overwhelm the surface with various high frequencies, i.e. the shorter wavelength waves ride on the top of the low-frequency waves.

Figure 15 shows the phase and spectral relations at jet and the free surface for the three forcing accelerations of 106, 120 and $145 \mathrm{~m} \mathrm{~s}^{-2}$. The phase relations between the 
jet and the free surface are shown by the time series of the velocities for the first $4 \mathrm{~s}$. The three groups of data show that the maximum jet velocities always correspond to the minimum free surface velocities, i.e. the phase difference is always about $180^{\circ}$. Moreover, the characteristic frequencies of the jet and the free surface are the same, only with different peak values. Thus, the intensive interactions between the jet and the free surface are clearly confirmed by the data. Note that when the wall oscillates with a high frequency of $160 \mathrm{~Hz}$, the jets only oscillate with a low frequency of $3 \mathrm{~Hz}$. Therefore, the difference between the neighbour jets due to the opposite phases of the wall oscillation is $(1 / 160) \times(180 / 360) /(1 / 3) \times 360=3.4^{\circ}$ at most.

Figure 16 shows the phase relations for the jets from four antinodes at three forcing accelerations of 106, 120 and $145 \mathrm{~m} \mathrm{~s}^{-2}$. All four jets are seen to be phase locked with the free surface. They transfer energy from the wall to the free surface and eventually excite the low-frequency three-dimensional gravity waves.

The measurements show a frequency lock or phase lock between the vortex shedding, vortex oscillations and gravity waves. In addition, the external forcing can cause the gravity wave to grow significantly. Both of these phenomena are typical of self-excited wave-vortex resonance. The jet induces the periodic vortex shedding from the oscillatory wall boundary layer around the jet. While the vortex interacts with the free surface to excite the surface sloshing, i.e. one mode of the three-dimensional gravity waves. The shedding frequency then locks to the frequency of the three-dimensional gravity waves, leading to resonance. With further increases of the forcing acceleration, or as the forcing continues, the jet fluctuations become stronger, but the shedding frequency does not change noticeably until the whole system vibrates violently creating much noise. The surface vibrates violently but in an orderly manner with the amplitude of the three-dimensional gravity waves reaching heights of approximately $3-5 \mathrm{~cm}$. This kind of self-excited wave-vortex resonance is similar to the self-excited resonance of a vortex street investigated by Welsh, Stokes \& Parker (1984).

The circular and azimuthal surface capillary waves are always present during the entire excitation sequence. After the gravity wave has grown sufficiently, the vertical velocity caused by the superposition of the capillary wave on the low-frequency gravity waves became large enough to overcome the surface tension and viscosity effects resulting in droplet ejection during the rising phase of the surface wave which is suppressed during the down swing. Due to the interaction with the capillary waves, the droplet ejection is not a single column or a single drop, but a mass of splashing drops. Here again, though there is resonance involved, the external forcing frequency has no influence on this resonance. The external forcing is only a source to generate the streaming jets. Thus, the droplet ejection mechanism expressed here is a non-resonant modal coupling that involves short-wave and long-wave interactions.

\subsection{Non-resonant modal-coupling excitation mechanism}

The experiments show two different phenomena for the droplet splashing with highfrequency splashing without gravity waves as discussed in $\S 3$ and single droplet ejection and droplet splashing with the low-frequency gravity wave. Figure 17 shows a schematic of the second excitation mechanism. The high-frequency external wall forcing forms oscillatory wall boundary layers. These boundary layers produce circular and azimuthal capillary surface waves that generate local streaming jets injected into the interior as mentioned in $\S 5.1$ when the local jets from opposite sides are strong enough to interact with each other, they turn downwards to form four global streaming jets and strong global vortical regions. The vortical regions are unstable 

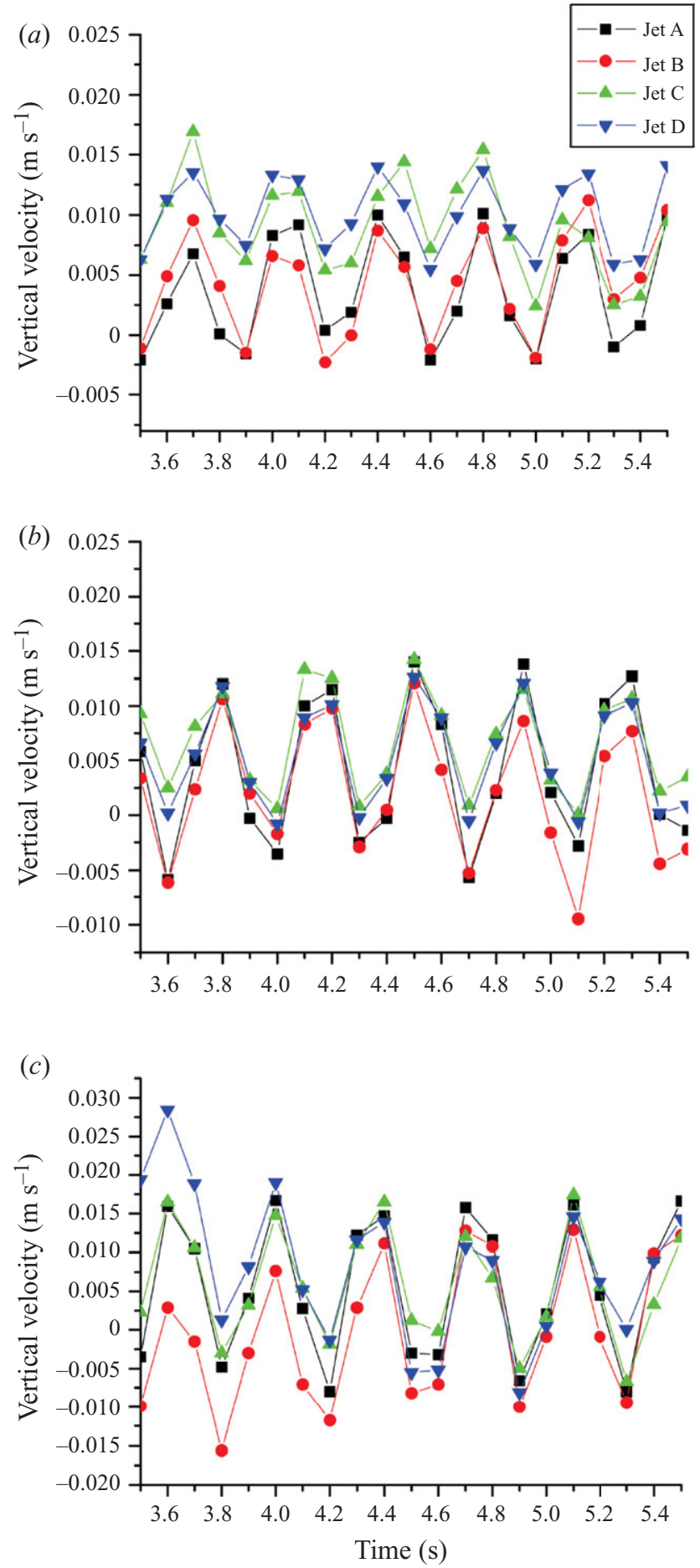

FIGURE 16. Phase relations of the four antinode jets at a forcing frequency of $160 \mathrm{~Hz}$ where $(a),(b)$ and $(c)$ correspond to forcing accelerations of 106,120 and $145 \mathrm{~m} \mathrm{~s}^{-2}$, respectively. 


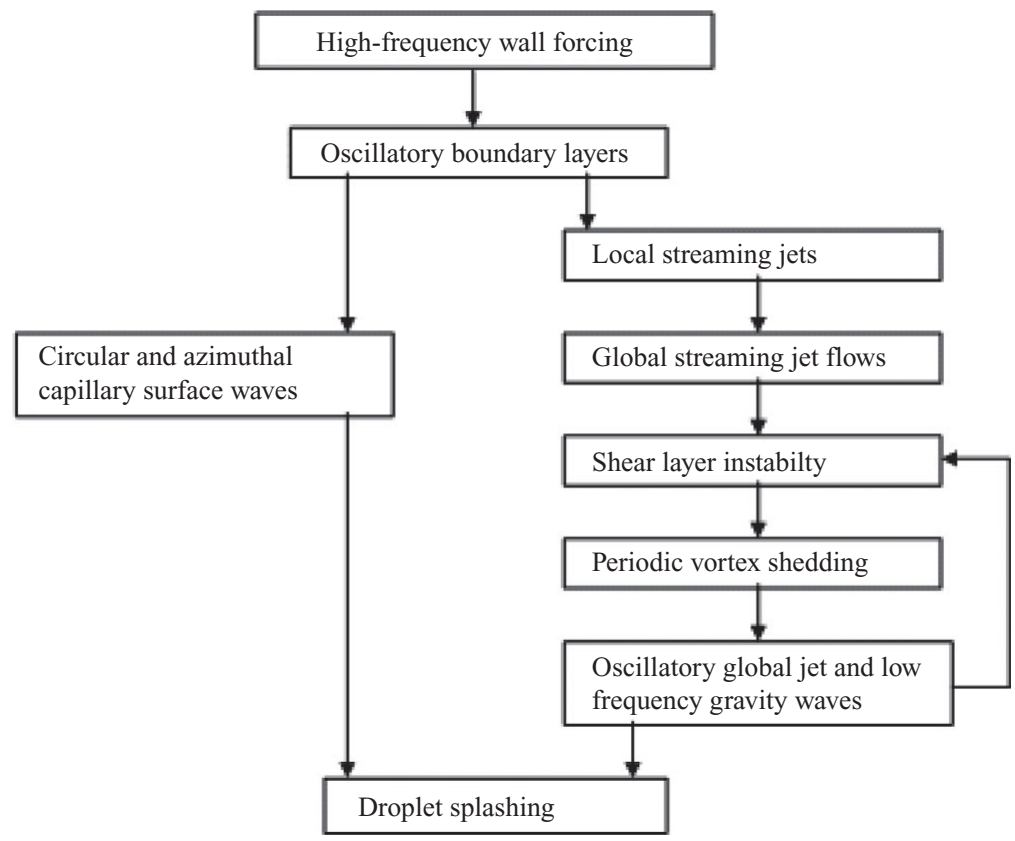

FIGURE 17. Schematic of possible excitation mechanisms for gravity wave generation and droplet splashing.

to small perturbations via the mechanism that leads to Kelvin-Helmholtz instability, which results in vortex shedding. The natural frequency, $f n$, of the periodic vortex shedding corresponds to the most unstable mode of the jets at the Strouhal number $S t=0.032$. Due to the finite size of the container, the periodic vortex-shedding then causes oscillations of the global jet in a feedback loop. When the four rows of phaselocked vortex streets meet in the centre of the basin, the flows in that area have to move up and down because of mass conservation, which causes the surface gravity wave. In these experiments, the vortex shedding frequency was found to lock-in with the natural frequency of the surface sloshing. The result is a positive feedback loop, which causes the low-frequency three-dimensional gravity waves to grow. Finally, the up-and-down motion of the large low-frequency gravity wave interacts with the high-frequency circular and azimuthal capillary waves to produce the droplet splashing. Furthermore, the interaction of the low-frequency gravity wave with the high-frequency capillary wave causes the ejected water to form a spray of droplets rather than a single water column.

\section{Discussion}

(i) 'Self-induced sloshing' in a tank was explained as a feedback loop between the interactions of the jet fluctuations and the pressure variations (Okamoto \& Hagiwara 1991; Fukaya et al. 1996 and Saeki et al. 2001). This is similar to the current excitation mechanism for wave-vortex resonate low-frequency three-dimensional gravity waves. However, the two phenomena are not the same with four vibration antinodes around the wall that provide the external forcing in the present experiments to induce four oscillatory jets from four different directions that interact with the surface waves. More importantly, the self-induced sloshing in those previous studies was caused by a 
pressure-driven jet injected into a two-dimensional tank with an outlet on the bottom. The present system has no inlet, so the mechanism described here are relevant to different set of engineering applications.

(ii) The PIV system was designed to only measure the fluorescence from seeded beads so as to eliminating the laser reflections from the wall to give satisfactory measurements of the curved moving interface. This system enabled measurements of the vorticity distributions not only in the jets but also on the water surface. If the liquid were just tap water, the free surface would have been contaminated and Marangoni effects would significantly modify the free surface mechanisms, as described by Jenkins \& Dysthe (1997), Hirsa, Lopez \& Miraghaie (2002) and many others. The surface tension would be affected by the impurities and the critical droplet velocity should differ from that for pure water. However, the critical droplet velocity is not the main issue in the present study, which focuses on the physical mechanism for the gravity wave formation and the droplet splashing generation.

(iii) This study shows that the two-dimensional analysis of Carlsson, Sen \& Löfdahl (2004) adequately models flows generated on vibrating surfaces so that it can be used to evaluate the flow effects of the surface vibrations. Our results also show that the normally neglected viscosity actually plays an important role for the short wave interactions.

Mui \& Dommermuth (1995) presented a two-dimensional numerical simulation of parasitic capillary waves generated by a steep viscous gravity-capillary wave to show that regions of high vorticity occur in the troughs of the parasitic capillary waves. Lin \& Perlin (2001) measured the velocity fields beneath the gravity-capillary waves and calculated their vorticity fields. The viscosity was found to indeed play an important role on the vorticity creation at the boundaries, on the vorticity diffusion from the surface to the interior and on the vorticity dissipation. More quantitative analyses of the viscous effects are needed with comparisons to the vorticity theories of $\mathrm{Wu}$ and his colleagues (Wu 1995; Wu et al. 2006) to clarify these effects.

\section{Conclusions}

(i) Experimental measurements of flows driven by vibrations of a cylindrical basin show that the forced surface waves and the flow inside the fluid bulk are rather complex. The PIV measurements of the flows, which were seeded with fluorescent particles to eliminate laser reflections from the wall, gave satisfactory measurements of the curved moving interface. This system enables measurements of the vorticity distributions not only in the jets but also on the water surface.

(ii) This paper describes the dragon wash phenomenon with a simple model that explains how the high-frequency sidewall forcing creates a low-frequency gravity wave, which leads to droplet splashing. Initially, capillary waves are created at the excitation frequency and then azimuthal waves appear that vibrate at half of the forcing frequency. As the excitation intensity increases, four jet-like structures, similar to streaming jets from a loudspeaker, simultaneously propagate from the boundary to the centre. As the jets from opposite walls interact with each other, the four jets are redirected downwards, so that the flow involves a large portion of the container. This produces maximum upward induced velocities at the four strongest vibrating points near the wall. Jet flow instabilities then create vortex shedding with frequencies that correlate well with the modified Strouhal number suggested by Ho \& Huerre (1984). The limited space in the container causes vortex-shedding feedback to the four jets so that the jets begin oscillating. The oscillating jets then interact with the water 
in the container to cause surface gravity waves. When the superposition of the upward gravity wave on the capillary waves becomes greater than a critical value that overcomes the surface tension, droplets are ejected. The interaction of the gravity wave with the high-frequency capillary waves causes the ejected water to form a spray of droplets rather than a single water column.

We acknowledge helpful comments by Professor D. J. Wang. This work was supported by the National Natural Science Foundation of China under Grant No. 10910301062 and the National Foundation for Distinguished Young Scholar of China under Grant No. 10525208. This work was also supported by the National Climb-B Plan under Grant No. 2009CB724100 and the National Natural Science Funds for Distinguished Young Scholar group under Grant No. 10921202.

\section{REFERENCES}

Carlsson, F., Sen, M. \& Löfdahl, L. 2004 Steady streaming due to vibrating walls. Phys. Fluids 16, 2375-2395.

Chang, C. C. 1999 Nonlinear theories of forced surface waves in a circular basin. PhD thesis, University of Wisconsin-Madison, Madison, WI.

Chang, C. C. \& Shen, M. C. $2000 a$ Chaotic motion and internal resonance of forced surface waves in a water-filled circular basin. Wave Motion 31, 317-331.

Chang, C. C. \& Shen, M. C. $2000 b$ Nonlinear capillary-gravity waves under an edge condition. J. Engng Math. 38, 391-402.

Davidson, B. J. \& Riley, N. 1972 Jets induced by oscillatory motion. J. Fluid Mech. 53, 287-303.

Faltinsen, O. M., Rognebakke, O. F. \& Timokha, A. N. $2006 a$ Resonant three-dimensional nonlinear sloshing in a square-base basin. Part 3. Base ratio perturbations. J. Fluid Mech. 551, 93-116.

Faltinsen, O. M., Rognebakke, O. F. \& Timokha, A. N. $2006 b$ Transient and steady-state amplitudes of resonant three-dimensional sloshing in a square-based tank with a finite fluid depth. Phys. Fluids 18, 012103.

FARADAY, M. 1831 On a peculiar class of acoustical figures; and on certain forms assumed by groups of particles upon vibrating elastic surfaces. Philos. Trans. R. Soc. Lond. 121, 299-340.

Fukaya, M., Madarame, H. \& OKamoto, K. 1996 Growth mechanism of self-induced sloshing caused by jet in rectangular tank (2nd report, multimode sloshing caused by horizontal rectangular jet). Trans. JSME, (B) 62, 64-71.

Gavrilyuk, I., Lukovsky, I. \& TiмокhA, A. 2004 Two-dimensional variational vibroequilibria and Faraday's drops. Z. Angew. Math. Phys. 55, 1015-1033.

Goodridge, C. L., Shi, W. T. \& Lathrop, D. P. 1996 Threshold dynamics of singular gravitycapillary waves. Phys. Rev. Lett. 76, 1824-1827.

Hirsa, A. H., Lopez, J. M. \& Miraghaie, R. 2002 Determination of surface shear viscosity via deep-channel flow with inertia. J. Fluid Mech. 470, 135-149.

Ho, C. M. \& Huerre, P. 1984 Perturbed free shear layers. Annu. Rev. Fluid Mech. 16, 365-424.

Hsien, D. Y. 1994 Standing water waves in a circular basin. In Proceedings of the International Conference on Hydrodynamics, pp. 74-79.

Hsien, D. Y. 1997 Water waves in an elastic vessel. Acta Mechanica Sin. 13, 289-303.

HsieH, D. Y. 2000 Theory of water waves in an elastic vessel. Acta Mechanica Sin. 16, 97-112.

Hsien, D. Y. \& Denissenko, P. 1998 Water waves in a circular elastic vessel: the experiment. Tech. Rep. 98-6-1. Department of Mathematics, Hong Kong University of Science and Technology.

Hu, H., Kobayashi, T., Saga, T., Segawa, T. N., Taniguchi, N. M. \& Okamoto, K. 1999 A PIV study on the self-induced sloshing in a tank with circulating flow. In Second Pacific Symposium on Flow Visualization and Image Processing, CD-ROM paper No. PF152.

Huntley, I. 1972 Observations on a spatial-resonance phenomenon. J. Fluid Mech. 53, 209-216.

Jenkins, A. D. \& Dysthe, K. B. 1997 The effective film viscosity coefficients of a thin floating fluid layer. J. Fluid Mech. 344, 335-337.

LeE, C. B. \& Wu, J. Z. 2008 Transition in wall-bounded flow. Appl. Mech. Rev. 61, 030802. 
Lighthill, M. J. 1978 Acoustic streaming. J. Sound Vib. 61, 391-418.

Lin, H. J. \& Perlin, M. 2001 The velocity and vorticity fields beneath gravity-capillary waves exhibiting parasitic ripples. Wave Motion 33, 245-257.

LiU, X. J., LIU, G. Y., JiA, Q. F. \& ZhaNG, S. X. 2005 Vibration analysis of the revolving gravity waves in fluid-filled cylindrical shells. J. Tianjin Univ. Sci. Techol. 38, 288-293.

Mahony, J. J. \& Sмith, R. 1972 On a model representation for the spatial-resonance phenomena. J. Fluid Mech. 53, 193-207.

Miles, J. \& Henderson, D. 1990 Parametrically forced surface waves. Annu. Rev. Fluid Mech. 22, $143-165$.

Mui, R. C. Y. \& Dommermuth, D. G. 1995 The vortical structure of parasitic capillary waves. J. Fluid Engng 117, 355-361.

Okamoto, K., Madarame, H. \& Hagiwara, T. 1991 Self-induced oscillation of free surface in a tank with circulating flow. In Proc. Fifth Intl Conf. Flow Induced Vibrations (ed. B. L. Clarkson), pp. 539-545. IMechE.

Peng, H. W. \& LeE, C. B. 2009 Periodic tripling and jet eruption of forced steep gravity waves. Mod. Phys. Lett. B 23, 397-400.

Peng, H. W., LI, R. Q., Chen, S. Z. \& LeE, C. B. 2008 Correlation dimension analysis and capillary wave turbulence in dragon-wash phenomena. Chin. Phys. B 17, 637-643.

Peng, H. W., Wang, D. J. \& Lee, C. B. 2005 Nonlinear low frequency water waves in a cylindrical shell. Mod. Phys. Lett. B 19, 1615-1618.

Peng, H. W., Yuan, H. J., Chen, S. Z., Wang, D. J. \& Lee, C. B. 2006 Experimental studies on dragon wash phenomena. J. Hydrodyn. 18, 507-510.

RAYLEIGH, L. 1883 On the circulation of air observed in Kundts tubes and some allied acoustical problems. Philos. Trans. R. Soc. Lond. Ser. A 175, 1-21.

RiLey, N. 2001 Steady streaming. Annu. Rev. Fluid Mech. 33, 43-65.

Royon-Lebeaud, A. Hopfinger, E. J. \& Cartellier, A. 2007 Liquid sloshing and wave breaking in circular and square-base cylindrical containers. J. Fluid Mech. 577, 467-494.

Saeki, S., Madarame, H. \& Окамото, K. 2001 Self-induced sloshing excited by a horizontally injected plane jet. J. Fluid Mech. 448, 81-114.

Saeki, S., Madarame, H., Okamoto, K. \& Tanaka, N. 1997 Numerical study on the self-induced sloshing. In FEDSM97-3401 ASME FED Summer Meeting.

Sharipov, F. M. \& Kremer, G. M. 1999 Non-isothermal Couette flow of a rarefied gas between two rotating cylinders. Eur. J. Mech. B/Fluids 18, 121-130.

Shen, M. C. \& HsieH, D. Y. 1993 Forced capillary-gravity waves in a circular basin. Wave Motion 18, 401-412.

Shen, M. C. \& YEH, N. S. 1997 Exact solution for forced capillary-gravity waves in a circular basin under Hocking's edge condition. Wave Motion 26, 117-126.

Sun, S. M., Shen, M. C. \& Hsien, D. Y. 1995 Nonlinear theory of forced surface waves in a circular basin. Wave Motion 21, 331-341.

Takizawa, A., Koshizuka, S. \& Kondo, S. 1992 Generalization of physical components boundary fitted coordinate (PCBFC) method for the analysis of free surface flow. Intl J. Numer. Meth. Fluids 15, 1213-1237.

Tower, D. P., Towers, C. E., Buckberry C. H. \& Reeves, M. 1999 A colour PIV system employing fluorescent particles for two-phase flow measurements. Meas. Sci. Technol. 10, 824-830.

WANG, D. J. 1993 Study on mechanical characteristics of ancient cultural relics. Sci. Conserv. Archaeol. 5, 35-39.

WANG, D. J. 2005 Bell chime, dragon washbasin - modern scientific information hidden in ancient Chinese science and technology. Technishe Mechanik 25, 9-16.

WeI, Q., WANG, D., YAn, B., Du, X. \& Chen, J. 1997 A visualization study on water spray of dragon washbasin. In Atlas of Visualization (ed. The Visualization Society of Japan), pp. 169-179. CRC press.

Welsh, M. C., Stokes, A. N. \& Parker, R. 1984 Flow-resonant sound interaction in a duct containing a plate. Part 1. Semicircular leading edge. J. Sound Vib. 95, 305-323.

WU, J. Z. 1995 A theory of three-dimensional interfacial vorticity dynamics. Phys. Fluids 7, 23752395.

Wu, J. Z., Ma, H. Y. \& Zhou, M. D. 2006 Vorticity and Vortex Dynamics. Springer-Verlag. 